\title{
Effectiveness of Structural Restrictions for Hybrid CSPs
}

\author{
Vladimir Kolmogorov ${ }^{\dagger}$ \\ vnk@ist.ac.at
}

Michal Rolínek ${ }^{\dagger}$
michal.rolinek@ist.ac.at

\author{
Rustem Takhanov $\ddagger$ \\ takhanov@mail.ru
}

\author{
${ }^{\dagger}$ Institute of Science and Technology Austria $\quad{ }^{\ddagger}$ Nazarbayev University, Kazakhstan
}

\begin{abstract}
Constraint Satisfaction Problem (CSP) is a fundamental algorithmic problem that appears in many areas of Computer Science. It can be equivalently stated as computing a homomorphism $\mathbf{R} \rightarrow \boldsymbol{\Gamma}$ between two relational structures, e.g. between two directed graphs. Analyzing its complexity has been a prominent research direction, especially for the fixed template CSPs where the right side $\boldsymbol{\Gamma}$ is fixed and the left side $\mathbf{R}$ is unconstrained.

Far fewer results are known for the hybrid setting that restricts both sides simultaneously. It assumes that $\mathbf{R}$ belongs to a certain class of relational structures (called a structural restriction in this paper). We study which structural restrictions are effective, i.e. there exists a fixed template $\boldsymbol{\Gamma}$ (from a certain class of languages) for which the problem is tractable when $\mathbf{R}$ is restricted, and NP-hard otherwise. We provide a characterization for structural restrictions that are closed under inverse homomorphisms. The criterion is based on the chromatic number of a relational structure defined in this paper; it generalizes the standard chromatic number of a graph.

As our main tool, we use the algebraic machinery developed for fixed template CSPs. To apply it to our case, we introduce a new construction called a "lifted language". We also give a characterization for structural restrictions corresponding to minor-closed families of graphs, extend results to certain Valued CSPs (namely conservative valued languages), and state implications for (valued) CSPs with ordered variables and for the maximum weight independent set problem on some restricted families of graphs.
\end{abstract}

\section{Introduction}

The Constraint satisfaction problems (CSPs) and the valued constraint satisfaction problems (VCSP) provide a powerful framework for analysis of a large set of computational problems arising in propositional logic, combinatorial optimization, artificial intelligence, graph theory, scheduling, biology, computer vision etc. Traditionally CSP is formalized either as a problem of (a) finding an assignment of values to a given set of variables, subject to constraints on the values that can be assigned simultaneously to specified subsets of variables, or as problem of (b) finding a homomorphism between two finite relational structures $A$ and $B$ (e.g., two oriented graphs). These two formulations are polynomially equivalent under the condition that the input constraints in the first case or input relations in the second case are given by lists of their elements. Soft version of CSP, that is VCSP, generalizes the CSP by replacing crisp constraints with cost functions applied to tuples of variables. In the VCSP we require to find the maximum (or minimum) of a sum of cost functions applied to corresponding variables.

The CSPs have been the cutting edge research field of theoretical computer science since the 70s, and recently this interest has been expanded to VCSP. One of the themes that revealed rich logical and algebraic structure of the CSPs was the question of classification of the problem's computational 
complexity when constraint relations are restricted to a given set of relations or, alternatively, when the second relational structure is some fixed $\boldsymbol{\Gamma}$. Thus, this problem is parameterized by $\boldsymbol{\Gamma}$, denoted as $\operatorname{CSP}(\boldsymbol{\Gamma})$ and called a fixed template CSP with a template $\boldsymbol{\Gamma}$ (another name is a non-uniform CSP). E.g., if the domain set is boolean and $\boldsymbol{\Gamma}$ is a relational structure with four ternary predicates $x \vee y \vee z$, $\bar{x} \vee y \vee z, \bar{x} \vee \bar{y} \vee z, \bar{x} \vee \bar{y} \vee \bar{z}, \operatorname{CSP}(\boldsymbol{\Gamma})$ models 3-SAT which is historically one the first NP-complete problems [12]. At the same time, if we restrict $\boldsymbol{\Gamma}$ to binary predicates, then we obtain tractable 2-SAT. Generally, Schaeffer proved [30] that for any template $\boldsymbol{\Gamma}$ over the boolean set, $\operatorname{CSP}(\boldsymbol{\Gamma})$ is either in $\mathrm{P}$ or NP-complete, and any tractable constraint language belongs to one of 6 classes (0 or 1preserving, binary, horn, anti-horn and linear subspaces). When $\boldsymbol{\Gamma}$ contains only one graph (irreflexive symmetric predicate) Hell and Nešetřil [20] proved an analogous statement, by showing that only for bipartite graphs the problem is tractable. Feder and Vardi [15] found that all fixed template CSPs can be expressed as problems in a fragment of SNP, called Monotone Monadic SNP (MM SNP). They introduced this class as a natural restriction of SNP for which Ladner's argument about the existence of problems with intermediate complexity between $\mathrm{P}$ and NP-hard could not be applied. Moreover, they showed that all problems in MM SNP can be reduced with respect to Turing reduction to fixed template CSPs and, thus, non-uniform CSPs complexity classification would lead to a classification of MM SNP problems. This result placed fixed-template CSPs into a broad logical context that naturally lead to a conjecture that such CSPs are either tractable or NP-hard, the so called dichotomy conjecture.

In 22] Jeavons observed that any predicate given by primitive positive formula using predicates of the template $\boldsymbol{\Gamma}$, when added to $\boldsymbol{\Gamma}$, does not change the complexity of $\operatorname{CSP}(\boldsymbol{\Gamma})$. This result clarified that the computational complexity of $\operatorname{CSP}(\boldsymbol{\Gamma})$ is fully defined by the minimal predicate clone that contains predicates of $\boldsymbol{\Gamma}$. In universal algebra, it has long been known that the predicate clones are dual to the so called functional clones [29, 25, 16]. Specifically, it implies that the complexity of $\operatorname{CSP}(\boldsymbol{\Gamma})$ is defined by the set of polymorphisms of $\boldsymbol{\Gamma}$. The last was the main motive for subsequent research. Intensive studies in this direction lead to a conjectured algebraic description of all tractable templates made by Bulatov, Jeavons, and Krokhin [7], with subsequent reformulations of this conjecture by Maroti and McKenzie [27]. In the long run it was shown by Siggers [31] that if Bulatov-JeavonsKrokhin characterization of tractable templates is correct, then the tractable core structures can be characterized as those that admit a single 6-ary polymorphism that satisfies a certain equality. The last fact will serve as a key ingredient for one of our results.

Besides fixed template CSPs, another parameterization of CSP concerns restrictions on the left relational structure of the input. If we restrict the left structure of the input to some specified set $\mathcal{H}$ and impose no restriction on the right relational structure, then the problem is called CSP with structural restrictions $\mathcal{H}$. For example, if $\mathcal{H}$ is a set of graphs with treewidth less or equal to $k \in \mathbb{N}$, then the problem can be solved in polynomial time. It was found by Grohe [18] that any structural restriction $\mathcal{H}$ that defines tractable CSP should be of bounded treewidth modulo homomorphic equivalence.

Related work. Since many (V)CSP instances do not fall into any of the tractable classes offered by one of the previous approaches, there has been growing interest in the so-called hybrid restrictions. That is when the input is restricted to a subset of all input pairs $(\mathbf{R}, \boldsymbol{\Gamma})$. One approach to this problem is to construct a new structure for any input $(\mathbf{R}, \boldsymbol{\Gamma}), G_{\mathbf{R}, \boldsymbol{\Gamma}}$, and shift the analysis to $G_{\mathbf{R}, \boldsymbol{\Gamma}}$. In case of binary CSPs (i.e. when all predicates of an input are binary) it is natural to define $G_{\mathbf{R}, \Gamma}$ as a microstructure graph 23 of a template $(\mathbf{R}, \boldsymbol{\Gamma})$. Thereby, a set of inputs for which certain local substructures in $G_{\mathbf{R}, \boldsymbol{\Gamma}}$ are forbidden form a parametrized problem. Cooper and Živný [13] investigated this formulation and found examples of specific forbidden substructures that result in tractable hybrid CSPs. Microstructure graphs also naturally appear in the context of fixed template CSPs. Specifically, all templates $\boldsymbol{\Gamma}$ with binary predicates that define fixed template CSPs for which local consistency preprocessing of the input results in a perfect microstructure graph were completely classified in [33]. 
Our results. The main topic of our paper is a hybrid framework for (V)CSP, when left structures are restricted to some set $\mathcal{H}$ and combined with a fixed right structure $\boldsymbol{\Gamma}$ (corresponding CSP is denoted as $\left.\operatorname{CSP}_{\mathcal{H}}(\boldsymbol{\Gamma})\right)$. The difficulty of applying known algebraic machinery to this framework is due to the fact that the closure operator, analogous to the minimal containing clone, cannot depend on $\boldsymbol{\Gamma}$ only. Therefore, in an algebraic theory of hybrid CSPs an analogue of primitive positive formula should depend on both input structures. In our approach we define for any $\mathbf{R} \in \mathcal{H}$ and $\boldsymbol{\Gamma}$ a set of predicates $\boldsymbol{\Gamma}_{\mathbf{R}}$ that we call a "lifted" language (see Sec. 5 ). Our key idea is that the closures $\left\langle\boldsymbol{\Gamma}_{\mathbf{R}}\right\rangle$ for $\mathbf{R} \in \mathcal{H}$, under certain conditions, could maintain the information on the tractability of $\operatorname{CSP}_{\mathcal{H}}(\boldsymbol{\Gamma})$. In this paper, by that "certain conditions" we understand the property that $\mathcal{H}$ is closed under inverse homomorphisms. We are especially interested in a classification of structural restrictions $\mathcal{H}$ closed under inverse homomorphisms for which we could find a template $\boldsymbol{\Gamma}$ (in a certain class of templates $\mathcal{C})$ that defines tractable $\operatorname{CSP}_{\mathcal{H}}(\boldsymbol{\Gamma})$, whereas a $\operatorname{CSP}(\boldsymbol{\Gamma})$ is NP-hard. We call such restrictions effective for a class $\mathcal{C}$. Our key results are formulated for 2 cases: the class of BJK languages, that is, the class of templates that are either tractable or have core a without a Siggers polymorphism, and a class of conservative valued templates.

Specifically, we prove that if $\mathcal{H}$ is a set of binary structures closed under inverse homomorphisms, it is effective for BJK languages if and only if $\{\chi(\mathbf{R}) \mid \mathbf{R} \in \mathcal{H}\}$ is bounded, where $\chi(\mathbf{R})$ is a chromatic number of $\mathbf{R}$ (considered as a graph). The last result is extended to the nonbinary case, with natural generalization of the chromatic number to arbitrary relational structures. A notable corollary of this result is that the set of acyclic digraphs is an ineffective structural restriction for BJK languages. This explains why NP-hardness arguments for certain fixed templates of digraph homomorphism problem can be extended to a case when the input digraph is acyclic [32]. Less straightforward corollary: let $\mathcal{H}$ be a set of binary structures such that their "graph copies" forbid specific minors, then $\mathcal{H}$ is effective for BJK languages if and only if $\{\chi(\mathbf{R}) \mid \mathbf{R} \in \mathcal{H}\}$ is bounded. The last statement does not require that $\mathcal{H}$ is closed under inverse homomorphisms.

For $\operatorname{VCSP}_{\mathcal{H}}(\boldsymbol{\Gamma})$ we prove an analogue of our previous result for a class $\mathcal{C}$ of all conservative valued templates. We obtain as a corollary that the maximum weight independent set problem is still NP-hard in some graph classes.

Organization. In Sec. 2 we give all the preliminary definitions and state theorems that we need. In Sec. 3 we state our main results (Theorems 22, 23 and 26) and their implications. The proofs of the main theorems are given in Sec. 447.

\section{Preliminaries}

Throughout the paper we assume $P \neq N P$. A problem is called tractable if it can be solved in polynomial time.

The symbol $[n]$ will denote the set $\{1, \ldots, n\}$, and $\overline{\mathbb{Q}}=\mathbb{Q} \cup\{\infty\}$ the set of rational numbers with (positive) infinity. Also $D$ will stand for a finite set.

We will denote the tuples in lowercase boldface such as $\mathbf{a}=\left(a_{1}, \ldots, a_{k}\right)$. Also for mappings $h: A \rightarrow$ $B$ and tuples $\mathbf{a}=\left(a_{1}, \ldots, a_{k}\right)$, where $a_{j} \in A$ for $j=1, \ldots, k$, we will write $\mathbf{b}=\left(h\left(a_{1}\right), \ldots, h\left(a_{k}\right)\right)$ simply as $\mathbf{b}=h(\mathbf{a})$. Relational structures will be denoted in uppercase boldface as $\mathbf{R}=\left(R, r_{1}, \ldots, r_{k}\right)$.

Finally let $\operatorname{ar}(\varrho), \operatorname{ar}(\mathbf{a})$, and $\operatorname{ar}(f)$ stand for arity of a relation $\varrho$, size of a tuple $\mathbf{a}$, and arity (number of parameters) of a function $f$, respectively.

\subsection{Fixed template CSP}

We will first formulate the general CSP in an algebraic way as a decision problems whether there exists a homomorphism between certain relational structures. 
Definition 1. Let $\mathbf{R}=\left(R, r_{1}, \ldots, r_{k}\right)$ and $\mathbf{R}^{\prime}=\left(R^{\prime}, r_{1}^{\prime}, \ldots, r_{k}^{\prime}\right)$ be relational structures with a common signature (that is $\operatorname{ar}\left(r_{i}\right)=\operatorname{ar}\left(r_{i}^{\prime}\right)$ for every $\left.i=1, \ldots, k\right)$. A mapping $h: R \rightarrow R^{\prime}$ is called a homomorphism from $\mathbf{R}$ to $\mathbf{R}^{\prime}$ if for each $i=1, \ldots, k$, whenever $\left(x_{1}, \ldots, x_{\operatorname{ar}\left(r_{i}\right)}\right) \in r_{i}$, then $\left(\left(h\left(x_{1}\right), \ldots, h\left(x_{\operatorname{ar}\left(r_{i}^{\prime}\right)}\right)\right) \in r_{i}^{\prime}\right.$. In that case, we write $\mathbf{R} \stackrel{h}{\rightarrow} \mathbf{R}^{\prime}$ or sometimes just $\mathbf{R} \rightarrow \mathbf{R}^{\prime}$.

Definition 2 (General CSP). The general CSP is the following decision problem. Given a pair of relational structures with common signature $\mathbf{R}=\left(V, r_{1}, \ldots, r_{k}\right)$ and $\boldsymbol{\Gamma}=\left(D, \varrho_{1}, \ldots, \varrho_{k}\right)$, decide whether $\mathbf{R} \rightarrow \boldsymbol{\Gamma}$. Equivalently, decide whether there is a mapping $h: V \rightarrow D$ that satisfies

$$
\bigwedge_{(\varrho, \mathbf{v}) \in T}[h(\mathbf{v}) \in \varrho]
$$

where $T=\left\{\left(\varrho_{i}, \mathbf{v}\right) \mid i \in[k], \mathbf{v} \in r_{i}\right\}$ specifies the set of constraints.

The set $V$ represents the set of variables and we will only consider $V$ finite, similarly $D$ is the domain set or the set of labels for variables. The relations $r_{1}, \ldots, r_{k}$ specify the tuples of $V$ constrained by relations $\varrho_{1}, \ldots, \varrho_{k}$, respectively.

As we mentioned in the introduction, one natural way to restrict the general CSP is to fix the constraint types. A finitary relational structure $\boldsymbol{\Gamma}=\left(D, \varrho_{1}, \ldots, \varrho_{k}\right)$ over a fixed finite domain $D$ will be called a constraint language. For such $\Gamma$ we will denote by $\Gamma$ (without boldface) the set of relations $\left\{\varrho_{1}, \ldots, \varrho_{k}\right\}$; with some abuse of terminology set $\Gamma$ will also be called a constraint language. (Note that both views are used in the literature).

Definition 3 (Fixed template CSP). Let $D$ be a finite set and $\boldsymbol{\Gamma}$ a constraint language over D. Then the decision problem $\operatorname{CSP}(\boldsymbol{\Gamma})$ is defined as follows: given a relational structure $\mathbf{R}=\left(V, r_{1}, \ldots, r_{k}\right)$ of the same signature as $\boldsymbol{\Gamma}$, decide whether $\mathbf{R} \rightarrow \boldsymbol{\Gamma}$.

We will usually write $\operatorname{CSP}(\Gamma)$ instead of $\operatorname{CSP}(\boldsymbol{\Gamma})$. Although there are multiple relational structures $\boldsymbol{\Gamma}$ that correspond to the same set $\Gamma$, it can be seen that all choices give equivalent problems; this justifies the notation $\operatorname{CSP}(\Gamma)$.

\subsection{Fixed template VCSP}

A more general framework operates with cost functions $f: D^{n} \rightarrow \overline{\mathbb{Q}}$ instead of relations $\varrho \subseteq D^{n}$. This idea leads to the notion of valued CSP.

Definition 4. We denote the set of all functions $f: D^{n} \rightarrow \overline{\mathbb{Q}}$ by $\boldsymbol{\Phi}_{D}^{(n)}$ and let $\boldsymbol{\Phi}_{D}=\bigcup_{n \geq 1} \boldsymbol{\Phi}_{D}^{(n)}$. We will often call the functions in $\boldsymbol{\Phi}_{D}$ cost functions over D. For every cost function $f \in \boldsymbol{\Phi}_{D}^{(n)}$, let $\operatorname{dom} f=\{x \mid f(x)<\infty\}$. Note that $\operatorname{dom} f$ can be considered both as an $n$-ary relation and as an $n$-ary function such that $\operatorname{dom} f(x)=0$ if and only if $f(x)$ is finite.

We will say that the cost functions in $\mathbf{\Phi}_{D}$ take values. Note that in some papers on VCSP, e.g. [11, 34, cost functions are called weighted relations.

Definition 5. An instance of the valued constraint satisfaction problem (VCSP) is specified by finite sets $D, V$ and a function from $D^{V}$ to $\overline{\mathbb{Q}}$ given by

$$
f_{\mathcal{I}}(h)=\sum_{(f, \mathbf{v}) \in T} w(f, \mathbf{v}) f(h(\mathbf{v}))
$$


where $V$ is a finite set of variables, $w(f, \mathbf{v})$ are positive numbers 1 and $T$ is a finite set of constraints of the form $(f, \mathbf{v})$ where $f \in \boldsymbol{\Phi}_{D}$ is a cost function and $\mathbf{v} \in V^{\operatorname{ar}(f)}$ is a tuple of variables of size $\operatorname{ar}(f)$. The goal is to find an assignment (or labeling) $h \in D^{V}$ that minimizes $f_{\mathcal{I}}$.

Note that $f_{\mathcal{I}}$ can also be looked at as a cost function over the variable set $V$.

Definition 6. $A$ valued constraint language over $D$ is either a tuple $\boldsymbol{\Gamma}=\left(D, f_{1}, \ldots, f_{k}\right)$ with $f_{1}, \ldots, f_{k} \in$ $\mathbf{\Phi}_{D}$ or the corresponding finite set $\Gamma=\left\{f_{1}, \ldots, f_{k}\right\} \subseteq \boldsymbol{\Phi}_{D}$. We will denote by $\operatorname{VCSP}(\Gamma)$ the class of all VCSP instances in which the cost functions are all contained in $\Gamma$.

This framework subsumes many other frameworks studied earlier and captures many specific wellknown problems, including $k$-Sat, Graph $k$-Colouring, Max Cut, Min Vertex Cover, and others (see [21]).

A function $f \in \boldsymbol{\Phi}_{D}^{(n)}$ that takes values in $\{0, \infty\}$ is called crisp. We will often view it as a relation in $D^{n}$, and vice versa (this should be clear from the context). If language $\Gamma$ is crisp (i.e. it contains only crisp functions), then $\operatorname{VCSP}(\Gamma)$ is a pure feasibility problem corresponding to $\operatorname{CSP}(\Gamma)$. Note, however, that according to our definitions there is a slight difference between the two: $\operatorname{CSP}(\Gamma)$ is a decision problem while $\operatorname{VCSP}(\Gamma)$ asks to compute a solution explicitly if it exists.

The dominant research line in this area is to classify the complexity of problems $\operatorname{VCSP}(\Gamma)$. Sometimes, problems $\operatorname{CSP}(\Gamma)$ and $\operatorname{VCSP}(\Gamma)$ are defined also for infinite languages $\Gamma$ and then $\operatorname{VCSP}(\Gamma)$ is called tractable if for each finite $\Gamma^{\prime} \subseteq \Gamma, \operatorname{VCSP}\left(\Gamma^{\prime}\right)$ is tractable. Also, $\operatorname{VCSP}(\Gamma)$ is called NP-hard if for some finite $\Gamma^{\prime} \subseteq \Gamma, \operatorname{VCSP}\left(\Gamma^{\prime}\right)$ is NP-hard. In turn, we will focus purely on finite languages $\Gamma$.

\subsection{Polymorphisms, Expressibility}

Let $\mathcal{O}_{D}^{(m)}$ denote the set of all operations $g: D^{m} \rightarrow D$ and let $\mathcal{O}_{D}=\bigcup_{m \geq 1} \mathcal{O}_{D}^{(m)}$. When $D$ is clear from the context, we will sometimes write simply $\mathcal{O}^{(m)}$ and $\mathcal{O}$.

Any language $\Gamma$ defined on $D$ can be associated with a set of operations on $D$, known as the polymorphisms of $\Gamma$, defined as follows.

Definition 7. An operation $g \in \mathcal{O}_{D}^{(m)}$ is a polymorphism of a cost function $f \in \boldsymbol{\Phi}_{D}$ if for any $\mathbf{x}^{1}, \ldots, \mathbf{x}^{m} \in \operatorname{dom} f$, we have that $g\left(\mathbf{x}^{1}, \ldots, \mathbf{x}^{m}\right) \in \operatorname{dom} f$ where $g$ is applied component-wise.

For any valued constraint language $\Gamma$ over a set $D$, we denote by $\operatorname{Pol}(\Gamma)$ the set of all operations on $D$ that are polymorphisms of every $f \in \Gamma$.

Clearly, if $g$ is a polymorphism of a cost function $f$, then $g$ is also a polymorphism of $\operatorname{dom} f$. For $\{0, \infty\}$-valued functions, which naturally correspond to relations, the notion of a polymorphism defined above coincides with the standard notion of a polymorphism for relations. Note that the projections, i.e. operations of the form $e_{n}^{i}\left(x_{1}, \ldots, x_{n}\right)=x_{i}$, are polymorphisms of all valued constraint languages. Polymorphisms play the key role in the algebraic approach to the CSP, but for VCSPs more general constructs are necessary. Now we define them.

Definition 8. An m-ary fractional operation $\omega$ on $D$ is a probability distribution on $\mathcal{O}_{D}^{(m)}$. The support of $\omega$ is defined as $\operatorname{supp}(\omega)=\left\{g \in \mathcal{O}_{D}^{(m)} \mid \omega(g)>0\right\}$.

\footnotetext{
${ }^{1}$ We will allow two possibilities: (i) weights are positive integers, and the length of the description of $\mathcal{I}$ grows linearly with $w(f, \mathbf{v})$; (ii) weights are positive rationals. All our statements for VCSPs will hold under both models. Note that in the literature weights $w(f, \mathbf{v})$ are usually omitted, and $T$ is allowed to be a multiset rather than a set; this is equivalent to model (i). Including weights will be convenient for hybrid VCSPs.
} 
Definition 9. A m-ary fractional operation $\omega$ on $D$ is said to be a fractional polymorphism of a cost function $f \in \mathbf{\Phi}_{D}$ if for any $\mathbf{x}^{1}, \ldots, \mathbf{x}^{m} \in \operatorname{dom} f$, we have

$$
\sum_{g \in \operatorname{supp}(\omega)} \omega(g) f\left(g\left(\mathbf{x}^{1}, \ldots, \mathbf{x}^{m}\right)\right) \leq \frac{1}{m}\left(f\left(\mathbf{x}^{1}\right)+\ldots+f\left(\mathbf{x}^{m}\right)\right) .
$$

For a constraint language $\Gamma, \operatorname{Pol}(\Gamma)$ will denote the set of all fractional operations that are fractional polymorphisms of each function in $\Gamma$. Also, let $\operatorname{Pol}^{+}(\Gamma)=\left\{g \in \mathcal{O}_{D} \mid g \in \operatorname{supp}(\omega), \omega \in \operatorname{fPol}(\Gamma)\right\}$.

Clearly, we have $\mathrm{Pol}^{+}(\Gamma) \subseteq \mathrm{Pol} \Gamma$ for any $\Gamma$.

The key observation in the algebraic approach to (V)CSP is that neither the complexity nor the algebraic properties of a language $\Gamma$ change when functions "expressible" from $\Gamma$ in a certain way are added to it.

Definition 10. For a constraint language $\Gamma$, let $\langle\Gamma\rangle$ denote the set of all functions $f\left(x_{1}, \ldots, x_{k}\right)$ such that, for some instance $\mathcal{I}$ of $\operatorname{VCSP}(\Gamma)$ with objective function $f_{\mathcal{I}}\left(x_{1}, \ldots, x_{k}, x_{k+1}, \ldots, x_{n}\right)$, we have

$$
f\left(x_{1}, \ldots, x_{k}\right)=\min _{x_{k+1}, \ldots, x_{n}} f_{\mathcal{I}}\left(x_{1}, \ldots, x_{k}, x_{k+1}, \ldots, x_{n}\right) .
$$

We then say that $\Gamma$ expresses $f$, and call $\langle\Gamma\rangle$ the expressive power of $\Gamma$.

Lemma $11([9,10])$. Let $f \in\langle\Gamma\rangle$. Then

(a) if $\omega \in \mathrm{fPol}(\Gamma)$, then $\omega$ is a fractional polymorphism of $f$ and of $\operatorname{dom} f$;

(b) $\operatorname{VCSP}(\Gamma)$ is tractable if and only if $\operatorname{VCSP}(\Gamma \cup\{f$, $\operatorname{dom} f\})$ is tractable;

(c) $\operatorname{VCSP}(\Gamma)$ is NP-hard if and only if $\operatorname{VCSP}(\Gamma \cup\{f, \operatorname{dom} f\})$ is NP-hard.

\subsection{Algebraic dichotomy conjecture}

The condition for tractability of CSPs was first conjectured by Bulatov, Krokhin, and Jeavons [7], and a number of equivalent formulations was later given in [31, 27, 2. We will use the formulation by Siggers [31; it will be important for our purposes that Siggers polymorphisms have a fixed arity six and so for example on a fixed finite domain $D$ there is only a finite number of them.

Definition 12. An operation $s: D^{6} \rightarrow D$ is called a Siggers operation on $D$ if for each $x, y \in D$ we have

$$
\begin{aligned}
& s(x, x, x, x, y, y)=s(x, y, x, y, x, x) \\
& s(y, y, x, x, x, x)=s(x, x, y, x, y, x) \\
& s(x, x, x, x, x, x)=x .
\end{aligned}
$$

The conjecture is usually stated for core languages. To reduce the number of definitions, we will give an alternative formulation that avoids cores. For a language $\Gamma$ on $D$ and a domain $D^{\prime} \subseteq D$ let $\Gamma\left[D^{\prime}\right]$ be the language obtained from $\Gamma$ by restricting each function to the domain $D^{\prime}$.

Definition 13. Tuple $(g, s)$ will be called a Siggers pair on a domain $D$ if $g$ is a unary operation on $D$ satisfying $g \circ g=g$ and $s$ is a Siggers operation on $g(D) \subseteq D$. We say that a crisp language $\Gamma$ on domain $D$ admits $(g, s)$ if $g$ is a unary polymorphism of $\Gamma$ and $s$ is a 6-ary polymorphism of $\Gamma[g(D)]$.

Theorem 14 ([31]). A crisp constraint language $\Gamma$ that does not admit a Siggers pair is NP-Hard. 
Conjecture 1 (A version of the Algebraic Dichotomy Conjecture). If a crisp language $\Gamma$ admits a Siggers pair, then $\operatorname{CSP}(\Gamma)$ is tractable.

There has been remarkable progress on this conjecture. It has been verified for domains of size 2 [30] and 3 [4], or for languages containing all unary relations on $D$ [5]. It has also been shown that it is equivalent to its restriction for directed graphs (that is when $\Gamma$ contains a single binary relation $\varrho$ ) [8]. Further, the conjecture holds if $\varrho$ corresponds to a directed graph with no sources and sinks [3]. Nevertheless, in the general case the conjecture remains open.

Definition 15. A crisp language $\Gamma$ is called a BJK language if it satisfies one of the following:

- $\operatorname{CSP}(\Gamma)$ is tractable

- $\Gamma$ does not admit a Siggers pair.

Conjecture 2 (Another version of the Algebraic Dichotomy Conjecture). Every crisp language $\Gamma$ is a BJK language.

\subsection{Hybrid (V)CSP setting}

Definition 16. Let us call a family $\mathcal{H}$ of relational structures with a common signature a structural restriction. If all the relations in $\mathcal{H}$ are unary, we call $\mathcal{H}$ all-unary.

Definition 17 (Hybrid CSP). Let $D$ be a finite domain, $\boldsymbol{\Gamma}$ a constraint language over $D$, and $\mathcal{H}$ a structural restriction of the same signature as $\boldsymbol{\Gamma}$. We define $\operatorname{CSP}_{\mathcal{H}}(\boldsymbol{\Gamma})$ as the following decision problem: given a relational structure $\mathbf{R} \in \mathcal{H}$ as input, decide whether $\mathbf{R} \rightarrow \boldsymbol{\Gamma}$.

Definition 18 (Hybrid VCSP). Let $D$ be a finite domain, $\boldsymbol{\Gamma}=\left(D, f_{1}, \ldots, f_{k}\right)$ a valued constraint language over $D$, and $\mathcal{H}$ a structural restriction of the same signature as $\boldsymbol{\Gamma}$. We define $\operatorname{VCSP}_{\mathcal{H}}(\boldsymbol{\Gamma})$ as the class of instances of the following form.

An instance is a function from $D^{V}$ to $\overline{\mathbb{Q}}$ given by

$$
f_{\mathcal{I}}(h)=\sum_{(f, \mathbf{v}) \in T} w(f, \mathbf{v}) f(h(\mathbf{v})),
$$

where $V$ is a finite set of variables, $w(f, \mathbf{v})$ are positive numbers and $T$ is a finite set of constraints determined by some relational structure $\mathbf{R}=\left(V, r_{1}, \ldots, r_{k}\right) \in \mathcal{H}$ as follows: $T=\left\{\left(f_{i}, \mathbf{v}\right) \mid i \in[k], \mathbf{v} \in\right.$ $r_{i}$. The goal is to find an assignment (or labeling) $h \in D^{V}$ that minimizes $f_{\mathcal{I}}$.

Definition 19. A structural restriction $\mathcal{H}$ is called effective for a class of (valued) languages $\mathcal{C}$ if there is a language $\boldsymbol{\Gamma}$ with $\Gamma \in \mathcal{C}$, of the same signature as $\mathcal{H}$, such that $(\mathrm{V}) \operatorname{CSP}(\Gamma)$ is $N P$-Hard, whereas $(\mathrm{V}) \mathrm{CSP}_{\mathcal{H}}(\boldsymbol{\Gamma})$ is tractable.

$\mathcal{H}$ is called ineffective for $\mathcal{C}$ if for every $\Gamma$ with $\Gamma \in \mathcal{C}$, of the same signature as $\mathcal{H},(\mathrm{V}) \operatorname{CSP}(\Gamma)$ and $(\mathrm{V}) \mathrm{CSP}_{\mathcal{H}}(\boldsymbol{\Gamma})$ are either both tractable or both $N P$-hard.

Note, some structural restrictions could potentially be neither effective nor ineffective for a given $\mathcal{C}$ (since there exist intermediate complexity classes between NP-hard and tractable problems).

Example 1. Let us give some examples of effective restrictions for the class $\mathcal{C}$ of all crisp languages.

Let $\mathcal{H}$ be the set of $k$-colorable graphs for $k>2$. Note that $k$-colorable graphs are exactly those that map homomorphically to the complete graph $K_{k}$. Therefore for the language $\Gamma=\left\{\neq_{D}\right\}$ on domain $D$ with $|D|>2$, we get that $\mathrm{CSP}_{\mathcal{H}}(\boldsymbol{\Gamma})$ is tractable (with a constant time algorithm that outputs YES), whereas $\operatorname{CSP}(\Gamma)$ is NP-Hard.

Similarly, also restricting to the class of planar graphs or perfect graphs is effective, since planar graphs are 4-colorable [1], and for perfect graphs the GRAPH $k$-COLOURING problem is known to be solvable in polynomial time [19]. 


\section{Our Results}

Most of our results will apply to structural restrictions $\mathcal{H}$ that are up-closed.

Definition 20. A family of relational structures $\mathcal{H}$ is called closed under inverse homomorphisms (or up-closed for short) if whenever $\mathbf{R}^{\prime} \rightarrow \mathbf{R}$ and $\mathbf{R} \in \mathcal{H}$, then also $\mathbf{R}^{\prime} \in \mathcal{H}$.

As examples of up-closed relational structures, let us mention directed acyclic graphs or $k$-colorable graphs. The proofs are straightforward. On the other hand, many natural graph classes do not possess this property, e.g. planar graphs and perfect graphs.

We introduce a notion of a chromatic number of relational structures that generalizes the usual chromatic number of graphs.

Definition 21. Let $\mathbf{R}=\left(V, r_{1}, \ldots, r_{k}\right)$ be a relational structure. A coloring of $\mathbf{R}$, that is a mapping $c: V \rightarrow[m]$, is improper if there is a color $j \in[m]$ such that for each $i \in[k]$, the relation $r_{i}$ contains a monochromatic tuple of the color $j$. A coloring that is not improper is called proper.

We define the chromatic number $\chi(\mathbf{R})$ of $\mathbf{R}$ to be the smallest number of colors that can yield a proper coloring of $\mathbf{R}$. (If no proper coloring exists, we set $\chi(\mathbf{R})=\infty$; this will happen if e.g. $\mathbf{R}$ contains only one unary relation). Also, we define the chromatic number $\chi(\mathcal{H})$ of a structural restriction as

$$
\chi(\mathcal{H})=\sup \{\chi(\mathbf{R}): \mathbf{R} \in \mathcal{H}\} .
$$

Theorem 22. A structural restriction $\mathcal{H}$ with $\chi(\mathcal{H})<\infty$ that is not all-unary is effective for the class of BJK languages.

Theorem 23. An up-closed structural restriction $\mathcal{H}$ with $\chi(\mathcal{H})=\infty$ is ineffective for the class of BJK languages.

In particular, Theorem 23 means that the Algebraic Dichotomy Conjecture would imply that upclosed structural restrictions $\mathcal{H}$ with $\chi(\mathcal{H})=\infty$ are ineffective for the class of all CSP languages. Next, we state our results for valued languages.

Definition 24. A valued language is called conservative if it contains all unary $\{0,1\}$-valued cost functions.

Definition 25. We say that a relational structure $\mathcal{H}$ does not restrict unaries if for each $\mathbf{R} \in \mathcal{H}$ of the form $\mathbf{R}=\left(V, r_{1}, \ldots, r_{i-1}, r_{i}, r_{i+1}, \ldots, r_{k}\right)$ with $\operatorname{ar}\left(r_{i}\right)=1$ and for each unary relation $r_{i}^{\prime} \subseteq V$, we have $\mathbf{R}^{\prime} \in \mathcal{H}$, where $\mathbf{R}^{\prime}=\left(V, r_{1}, \ldots, r_{i-1}, r_{i}^{\prime}, r_{i+1}, \ldots, r_{k}\right)$.

Theorem 26. An up-closed structural restriction $\mathcal{H}$ with $\chi(\mathcal{H})=\infty$ that does not restrict unaries is ineffective for the class of conservative valued languages.

Remark 1. Note that our current techniques do not easily extend to other classes of VCSPs, e.g. finitevalued languages [35]. Informally, the difficulty can be attributed to the fact that tractable finite-valued languages are characterized by fractional polymorphisms with an arbitrarily large support (if the size of the domain is not fixed), whereas for conservative languages we need two fractional polymorphisms that contain a constant number of operations in the support, namely 2 and 3 [24].

The proofs of the main theorems are described in the later sections. But first in Sec. 3.1 3.3 we will list three implications of our theorems. 


\subsection{Ordered CSP}

One natural structural restriction to fixed template CSP is to introduce ordering of variables and request the constraints to respect the ordering.

Definition 27. We call a relational structure $\left(V, r_{1}, \ldots, r_{k}\right)$ ordered if, after some identification of $V$ with $[n]$ for $n=|V|$, whenever $\left(v_{1}, \ldots, v_{\operatorname{ar}\left(r_{j}\right)}\right) \in r_{j}$ for some $j=1, \ldots, k$, then $v_{1}<\cdots<v_{\operatorname{ar}\left(r_{j}\right)}$.

Theorem 28. Let $\mathcal{H}$ be the set of all ordered relational structures of some fixed signature. Such structural restriction $\mathcal{H}$ is ineffective for BJK languages and for conservative valued languages.

Proof. It suffices to show that preconditions of Theorems 23 and 26 hold.

- $\mathcal{H}$ up-closed:

Let $\mathbf{R}=\left(V, r_{1}, \ldots, r_{k}\right) \in \mathcal{H}$ and $\mathbf{R}^{\prime} \rightarrow \mathbf{R}$ where $\mathbf{R}^{\prime}=\left(V^{\prime}, r_{1}^{\prime}, \ldots, r_{k}^{\prime}\right)$ and let $h: V^{\prime} \rightarrow V$ be the homomorphism. We may assume $V=[n]$. Let us define a partial order on $V^{\prime}$ such that $v_{1}^{\prime}<v_{2}^{\prime}$, if $h\left(v_{1}^{\prime}\right)<h\left(v_{2}^{\prime}\right)$. Extend this partial order arbitrarily to a total order on $[m]$ for $m=\left|V^{\prime}\right|$ and identify $V^{\prime}$ and $[m]$.

Now take $\left(v_{1}, \ldots, v_{\operatorname{ar}\left(r_{j}^{\prime}\right)}\right) \in r_{j}^{\prime}$ for some $j=1, \ldots, k$ and since $\left(h\left(v_{1}\right), \ldots, h\left(v_{\operatorname{ar}\left(r_{j}^{\prime}\right)}\right)\right) \in r_{j}$, we have $h\left(v_{1}\right)<\cdots<h\left(v_{\operatorname{ar}\left(r_{j}^{\prime}\right)}\right)$ and thus also $v_{1}<\cdots<v_{\operatorname{ar}\left(r_{j}^{\prime}\right)}$.

We have just verified that $\mathbf{R}^{\prime} \in \mathcal{H}$.

- $\chi(\mathcal{H})=\infty$ :

Fix $n \in \mathbb{N}$. We will construct $\mathbf{R} \in \mathcal{H}$ that cannot be properly colored with $n$ colors. Let $m$ be the maximal arity of relations in $\mathcal{H}$. Let $\mathbf{R}=\left(V, r_{1}, \ldots, r_{k}\right)$ where $V=[n(m-1)+1]$ and for $j=1, \ldots, k$ we set $\left(v_{1}, \ldots, v_{\operatorname{ar}\left(r_{j}\right)}\right) \in r_{j}$ if and only if $v_{1}<\cdots<v_{\operatorname{ar}\left(r_{j}\right)}$.

Clearly, $\mathbf{R} \in \mathcal{H}$. Now for any coloring with $n$ colors some color (say red) appears at least $m$ times. Let $v_{1}<\cdots<v_{m}$ be red elements of $V$. But then the tuples $\left(v_{1}, \ldots, v_{\operatorname{ar}\left(r_{j}\right)}\right)$ are red for $j=1, \ldots, k$ and hence the coloring is improper.

- $\mathcal{H}$ does not restrict unaries: this follows directly from the definitions.

This has an interesting consequence for graph homomorphism problems. Namely, restricting the input to directed acyclic graphs does not, assuming algebraic dichotomy conjecture, change the complexity of the problem.

Corollary 29. For the class of directed acyclic graphs $\mathcal{H}$, algebraic dichotomy conjecture implies that for every language $\boldsymbol{\Gamma}=(D, \varrho)$ with a binary relation $\varrho, \operatorname{CSP}(\Gamma)$ is tractable if and only if $\operatorname{CSP}_{\mathcal{H}}(\boldsymbol{\Gamma})$ is tractable.

Remark 2. A related result appeared in [15]. The authors showed that a dichotomy for CSPs with input structures restricted to partial orders gives the dichotomy for all CSPs. However, no connection is shown between $\operatorname{CSP}_{\mathcal{H}}(\boldsymbol{\Gamma})$ and $\operatorname{CSP}(\Gamma)$ as it is in our case. 


\subsection{Minor-closed families of graphs}

Theorem 30. Let the structural restriction $\mathcal{H}$ be a family of directed graphs such that the underlying family of undirected graphs is minor-closed. Then $\mathcal{H}$ is effective for BJK languages if and only if $\chi(\mathcal{H})<\infty$.

Proof. We use a result formulated as Lemma 2 in [28, that relies on an old theorem by Mader [26]: a minor-closed family of undirected graphs has either bounded chromatic number or contains all graphs.

In the first case we also have $\chi(\mathcal{H})<\infty$ and $\mathcal{H}$ is effective by Theorem 22. In the other case, for each $G=(V, E) \in \mathcal{H}$ and each pair $x, y \in V, x \neq y$, we have $(x, y) \in E$ or $(y, x) \in E$.

We will show that then $\mathcal{H}$ contains all directed acyclic graphs and thus is ineffective due to Theorem 23. In fact, it suffices to show that $\mathcal{H}$ contains a total order (a complete directed acyclic graphs) of every size, since every directed graph is a minor of some total order.

To this end, fix $n \in \mathbb{N}$ and pick $G \in \mathcal{H}$ with $R(n, n)$ vertices, where $R(n, n)$ is the corresponding Ramsey number. We set $V(G)=[R(n, n)]$ and color an edge $(x, y)$ blue if $x>y$ and red if $x<y$. By Ramsey's Theorem we are guaranteed to find a monochromatic clique of size $n$. This clique is a minor of $G$ and gives us the desired total order.

\subsection{Maximum Independent Set}

Although Theorem 26 is formulated for conservative languages, it also gives implications for some optimization problems corresponding to non-conservative languages. In this subsection, we will show that the classical problem of MAX WEIGHT INDEPENDENT SET is still intractable on some classes of graphs.

Given a class of undirected graphs $\mathcal{G}$, we write $\mathrm{MWIS}_{\mathcal{G}}$ to denote MAX WEIGHT INDEPENDENT SET problem (with positive node weights) restricted to class $\mathcal{G}$. If $\mathcal{G}$ is the class of all undirected graphs, let us write MWIS instead of $\mathrm{MWIS}_{\mathcal{G}}$. We say that $\mathcal{G}$ is up-closed if it satisfies the following condition: if $G, G^{\prime}$ are undirected graphs such that $G \in \mathcal{G}$ and $G^{\prime}$ maps homomorphically to $G$, then $G^{\prime} \in \mathcal{G}$.

Theorem 31. Let $\mathcal{G}$ be an up-closed family of undirected graphs with $\chi(\mathcal{G})=\infty$. Then $\mathrm{MWIS}_{\mathcal{G}}$ is NP-hard.

To prove this theorem, consider language $\boldsymbol{\Gamma}=\left(D, f, f_{1}, \ldots, f_{k}\right)$ where $D=\{0,1\}, f$ is the binary function with $f(1,1)=\infty$ and $f(0,0)=f(0,1)=f(1,0)=0$, and $\left\{f_{1}, \ldots, f_{k}\right\}$ is the set of all $\{0,1\}$ valued unary functions on $D$. Given a class of graphs $\mathcal{G}$, we define a structural restriction $\mathcal{H}(\mathcal{G})$ of the same signature as $\boldsymbol{\Gamma}$ that does not restrict unaries as follows:

$$
\mathcal{H}(\mathcal{G})=\left\{\left(V, G, V_{1}, \ldots, V_{k}\right): G \in \overrightarrow{\mathcal{G}}, V_{1}, \ldots, V_{k} \subseteq V=V(G)\right\}
$$

where $\overrightarrow{\mathcal{G}}$ denotes the family of all directed graphs that can be obtained by taking a graph $G \in \mathcal{G}$ and orienting edges in an arbitrary way.

Proposition 32. Let $\mathcal{G}$ be a family of undirected graphs closed under taking induced subgraphs. Then $\mathrm{MWIS}_{\mathcal{G}}$ and $\operatorname{VCSP}_{\mathcal{H}(\mathcal{G})}(\boldsymbol{\Gamma})$ are polynomial-time equivalent.

Proof. It will be convenient to treat MWIS $_{\mathcal{G}}$ as the MIN WEIGHT INDEPENDENT SET, where the weight of each node is a negative rational number. Clearly, this is equivalent to the original definition of MWIS $_{\mathcal{G}}$.

In one direction the reduction is trivial: any instance of $\mathrm{MWIS}_{\mathcal{G}}$ can be easily cast as an instance of $\operatorname{VCSP}_{\mathcal{H}(\mathcal{G})}(\boldsymbol{\Gamma})$ (assuming that vertices labeled with 1 correspond to vertices of an independent set). 
Let us consider the other direction. Let $\mathcal{I}$ be an instance of $\operatorname{VCSP}_{\mathcal{H}(\mathcal{G})}(\boldsymbol{\Gamma})$. Let $G=(V, E) \in \overrightarrow{\mathcal{G}}$ be the corresponding graph. After merging unary terms we can rewrite the objective function of $\mathcal{I}$ as

$$
f_{\mathcal{I}}(h)=\sum_{(u, v) \in E} w(u, v) f(h(u), h(v))+\sum_{v \in V} w_{v} h(u)+\text { const } \quad \forall h: V \rightarrow\{0,1\}
$$

where weights $w(u, v)$ are positive. Now set $V^{-}=\left\{v \in V: w_{v}<0\right\}$ and let $G^{-}$be the induced subgraph of $G$ on the vertex set $V^{-}$. Note that $G^{-} \in \overrightarrow{\mathcal{G}}$. Now solve the min weight independent set problem on $G^{-}$and label the chosen vertices with 1 and all others with 0 . It is easy to see that this is an optimal assignment for $\mathcal{I}$.

We can now prove Theorem 31. It can be checked that any up-closed class of graphs is closed under taking induced subgraphs, and so the precondition of Proposition 32 holds. Problem VCSP $(\Gamma)$ is polynomial-time equivalent to MWIS and thus is NP-hard. It is easy to check that up-closedness of $\mathcal{G}$ implies up-closedness of $\mathcal{H}(\mathcal{G})$. Therefore, by Theorem $26 \operatorname{VCSP}_{\mathcal{H}(\mathcal{G})}(\boldsymbol{\Gamma})$ is also NP-hard, and thus so is MWIS $_{\mathcal{G}}$ by Proposition 32 .

Remark 3. Let us mention that up-closed graph classes $\mathcal{H}$ with $\chi(\mathcal{H})=\infty$ can be non-trivial. Let $\mathcal{H}_{k}^{\text {odd }}$ be the class of graphs with odd girth at least $k$. Then for example $\mathcal{H}_{4}^{\text {odd }}$ is the class of trianglefree graphs. It can be checked that $\mathcal{H}_{k}^{\text {odd }}$ is up-closed for every $k$ (homomorphic image of an odd cycle contains an odd cycle of equal or smaller length). For the unbounded chromatic number we refer to a classical result [14 that states that the family of graphs $\mathcal{H}_{k}$ with girth at least $k$ has $\chi\left(\mathcal{H}_{k}\right)=\infty$. Since $\mathcal{H}_{k} \subseteq \mathcal{H}_{k}^{\text {odd }}$, we get also $\chi\left(\mathcal{H}_{k}^{\text {odd }}\right)=\infty$.

\section{Proof of Theorem 22}

We need to construct a BJK language $\boldsymbol{\Gamma}$ such that $\operatorname{CSP}(\Gamma)$ is $\mathrm{NP}$-Hard whereas $\operatorname{CSP}_{\mathcal{H}}(\boldsymbol{\Gamma})$ is polynomially tractable.

Let $n_{1}, \ldots, n_{k}$ be the arities of relational structures in $\mathcal{H}$. Also take $m$ such that $m \geq \chi(\mathcal{H})$ and $m>2$. We will define $\boldsymbol{\Gamma}=\left(D, \varrho_{1}, \ldots, \varrho_{k}\right)$ on the domain $D=D_{1} \cup \cdots \cup D_{k}$, where $D_{i}$ are pairwise disjoint copies of $[m]$. Also, let $d_{i}:[m] \rightarrow D$ such that $d_{i}(j)$ is the copy of $j$ in $D_{i}$. For $i \in[k]$ we set

$$
X_{\infty}^{i}=\left\{(a, \ldots, a):(a, \ldots, a) \subseteq D^{n_{i}}, a \in D_{i}\right\}
$$

and then define $\varrho_{i}=D^{n_{i}} \backslash X_{\infty}^{i}$.

- Hardness of $\operatorname{CSP}(\Gamma)$ : We will show that $\Gamma$ can express a certain coloring relation. Let us define a binary relation $\varrho \in\langle\Gamma\rangle$ as

$$
\varrho(x, y)=\left(\bigwedge_{i \in I_{1}}\left(\varrho_{i}(x) \wedge \varrho_{i}(y)\right)\right) \wedge\left(\bigwedge_{i \in I_{\geq 2}} \varrho_{i}(x, y, y, \ldots y)\right)
$$

where $I_{1}$ is the set of indices of the unary relations in $\Gamma$ and $I_{\geq 2}$ are the indices of the non-unary relations. Note that $I_{\geq 2}$ is nonempty. Let $D^{\prime}=\bigcup_{i \in I_{\geq 2}} D_{i}$, then $\left|D^{\prime}\right| \geq m$. It can be checked that $(x, y) \notin \varrho$ if $x \notin D^{\prime}$ or $y \notin D^{\prime}$. Finally, for $x, y \in D^{\prime}$, we clearly have $(x, y) \in \varrho$ if $x \neq y$ and also $(x, y) \notin \varrho$ for $x=y$, since for some $i \in I_{\geq 2}$ we have $(x, \ldots, x) \in X_{\infty}^{i}$. That is, $\varrho(x, y)$ corresponds to $\mathrm{a} \neq$ relation on $D^{\prime}$ (which corresponds to $\left|D^{\prime}\right|$-coloring) and since $\left|D^{\prime}\right| \geq m>2$ and $\varrho \in\langle\Gamma\rangle$ this makes $\mathrm{CSP}(\Gamma)$ NP-Hard by Lemma 11(c). 
- Tractability of $\operatorname{CSP}_{\mathcal{H}}(\boldsymbol{\Gamma})$ : We claim that a constant-time algorithm that outputs YES is correct for every instance of $\operatorname{CSP}_{\mathcal{H}}(\boldsymbol{\Gamma})$.

Consider an instance given by a relational structure $\mathbf{R}=\left(V, r_{1}, \ldots, r_{k}\right) \in \mathcal{H}$. Since $\chi(\mathbf{R}) \leq m$, there exists a proper coloring of $\mathbf{R}$ with colors $1, \ldots, m$. Now, as the coloring is proper, for each color class $j \in[m]$, there exists $i=i(j)$ such that the relation $r_{i}$ has no monochromatic tuple in the color $j$. Let us define a map $s: V \rightarrow D$. If $j$ is the color of $v \in V$, then let $s(v)=d_{i(j)}(j)$. We claim this assignment is feasible.

Indeed, suppose not, then there exist index $i \in[k]$ and a tuple $\mathbf{v}=\left(v_{1}, \ldots, v_{n_{k}}\right) \in r_{i}$ such that $s(\mathbf{v}) \notin \varrho_{i}$. Thus, $s(\mathbf{v})=(a, \ldots, a)$ for some $a \in D_{i}$. This means that $v_{1}, \ldots, v_{n_{k}}$ have the same color $j$ and $a=d_{i(j)}(j)$. Condition $d_{i(j)}(j) \in D_{i}$ implies that $i(j)=i$. We obtained that relation $r_{i}$ for $i=i(j)$ contains a monochromatic tuple in color $j$, which is a contradiction.

It remains to say that $\Gamma$ is a BJK language. First, observe that language $\{\varrho\}$ is a BJK language (binary relation $\rho$ corresponds to a digraph without sources and sinks, for which the Algebraic Dichotomy conjecture has been established in [3]). Since $\{\varrho\}$ is NP-hard, we obtain that $\rho$ does not admit a Siggers pair. By Lemma 33 below, $\Gamma$ also does not admit a Siggers operation, and thus is a BJK language.

Lemma 33. Let $\Gamma$ be a crisp language on a domain $D$ that admits a Siggers pair $(g, s)$ with $A=$ $g(D) \subseteq D$. Then language $\langle\Gamma\rangle$ also admits the Siggers pair $(g, s)$.

Proof. We need to show the following for every crisp function $f \in\langle\Gamma\rangle$ of arity $k=\operatorname{ar}(f)$ : (i) $f$ admits $g$ as a unary polymorpshism; (ii) $f_{\mid A}$ (the restriction of $f$ to $A^{k}$ ) admits $s$ as a 6-ary polymorphism. The first claim holds by Lemma 11(a). We will show that $f_{\mid A} \in\langle\Gamma[A]\rangle$, then the second claim will again follow by Lemma 11(a).

Since $f \in\langle\Gamma\rangle$, there exists a $\Gamma$-instance $\mathcal{I}$ with $n \geq k$ variables such that

$$
f(\mathbf{x})=\min _{\mathbf{y} \in D^{n-k}} f_{\mathcal{I}}(\mathbf{x}, \mathbf{y}) \quad \forall \mathbf{x} \in D^{k}
$$

Define a function $f^{\prime}: A^{k} \rightarrow\{0, \infty\}$ via

$$
f^{\prime}(\mathbf{x})=\min _{\mathbf{y} \in A^{n-k}} f_{\mathcal{I}}(\mathbf{x}, \mathbf{y}) \quad \forall \mathbf{x} \in A^{k}
$$

By construction, $f^{\prime} \in\langle\Gamma[A]\rangle$. It thus suffices to prove that $f_{\mid A}=f^{\prime}$. Consider $\mathbf{x} \in A^{k}$. Clearly, we have $f(\mathbf{x}) \leq f^{\prime}(\mathbf{x})$ (or equivalenty $\mathbf{x} \in \operatorname{dom} f^{\prime}$ implies $\mathbf{x} \in \operatorname{dom} f$ ). Suppose that $\mathbf{x} \in \operatorname{dom} f$. Then there exists $\mathbf{y} \in D^{n-k}$ such that $(\mathbf{x}, \mathbf{y}) \in \operatorname{dom} f_{\mathcal{I}}$. Since $g$ is a polymorphism of $f_{\mathcal{I}}$, we obtain $(g(\mathbf{x}), g(\mathbf{y})) \in \operatorname{dom} f_{\mathcal{I}}$. The properties of $g$ stated in Definition 13, in particular the idempotence, and the fact $x \in A^{k}$ give that $g(\mathbf{x})=\mathbf{x}$ and $g(\mathbf{y}) \in A^{n-k}$. Therefore, $\left(\mathbf{x}, \mathbf{y}^{\prime}\right) \in \operatorname{dom} f_{\mathcal{I}}$ for some $\mathbf{y}^{\prime} \in A^{n-k}$ and so $\mathbf{x} \in \operatorname{dom} f^{\prime}$.

\section{Constructing a "lifted" language}

For both Theorems 23 and 26 we need to show that tractability of the restricted problem implies tractability of the unrestricted one.

Let $\boldsymbol{\Gamma}=\left(D, f_{1}, \ldots, f_{k}\right)$ be a language of the same signature as $\mathcal{H}$ and $\mathbf{R}$ be a relational structure in $\mathcal{H}$. In this section we will construct a language $\Gamma_{\mathbf{R}}$ of finite size on a larger domain, based on $\boldsymbol{\Gamma}$ and $\mathbf{R}$. Our strategy will then be to link languages $\left\{\Gamma_{\mathbf{R}}: \mathbf{R} \in \mathcal{H}\right\}$, in terms of tractability, to both $\operatorname{VCSP}_{\mathcal{H}}(\boldsymbol{\Gamma})$ and $\operatorname{VCSP}(\Gamma)$. Namely, we will first prove the following. 
Proposition 34. Suppose that $\mathcal{H}$ is up-closed, $\mathbf{R} \in \mathcal{H}$ and $\boldsymbol{\Gamma}$ is a (valued) language. Then there is a polynomial-time reduction from $(\mathrm{V}) \operatorname{CSP}\left(\Gamma_{\mathbf{R}}\right)$ to $(\mathrm{V}) \operatorname{CSP}_{\mathcal{H}}(\boldsymbol{\Gamma})$. Consequently,

(a) if $(\mathrm{V}) \mathrm{CSP}_{\mathcal{H}}(\boldsymbol{\Gamma})$ is tractable, then so is $(\mathrm{V}) \mathrm{CSP}\left(\Gamma_{\mathbf{R}}\right)$;

(b) if $(\mathrm{V}) \operatorname{CSP}\left(\Gamma_{\mathbf{R}}\right)$ is NP-hard, then so is $(\mathrm{V}) \mathrm{CSP}_{\mathcal{H}}(\boldsymbol{\Gamma})$.

Using algebraic tools, we will then show in sections 6 and 7 how tractability of $\Gamma_{\mathbf{R}}$ for all $\mathbf{R} \in \mathcal{H}$ implies tractability of $\boldsymbol{\Gamma}$ for $\boldsymbol{\Gamma}$ lying in the particular language classes.

\subsection{Construction of $\Gamma_{\mathbf{R}}$}

Let us fix a relational structure $\mathbf{R}=\left(V, r_{1}, \ldots, r_{k}\right)$. For each $v \in V$ we create a unique copy of the domain $D$, and denote it $D_{v}$. We then define

$$
D_{\mathbf{R}}=\bigcup_{v \in V} D_{v}
$$

For $v \in V$ define a mapping $d_{v}: D \rightarrow D_{\mathbf{R}}$ such that $d_{v}(a)$ is the copy of $a$ in $D_{v}$. Also for tuples $\mathbf{a}=\left(a_{1}, \ldots, a_{p}\right) \in D^{p}$ and $\mathbf{v}=\left(v_{1}, \ldots, v_{p}\right) \in V^{p}$ we set $d_{\mathbf{v}}(\mathbf{a})=\left(d_{v_{1}}\left(a_{1}\right), \ldots, d_{v_{p}}\left(a_{p}\right)\right)$.

For the opposite direction, let $d(b)$ for $b \in D_{\mathbf{R}}$ be the natural projection of $b$ on $D$, and for a tuple $\mathbf{b}=\left(b_{1}, \ldots, b_{p}\right)$ let $d(\mathbf{b})=\left(d\left(b_{1}\right), \ldots, d\left(b_{p}\right)\right)$.

Now for a cost function $f \in \mathbf{\Phi}_{D}$ and $\mathbf{v} \in V^{\operatorname{ar}(f)}$ we will define a cost function on $D_{\mathbf{R}}$ of the same arity as $f$ via

$$
f^{\mathbf{v}}(\mathbf{x})=\left\{\begin{array}{ll}
f(\mathbf{y}) & \text { if } \mathbf{x}=d_{\mathbf{v}}(\mathbf{y}) \text { for some } \mathbf{y} \in D^{\operatorname{ar}(f)} \\
\infty & \text { otherwise }
\end{array} \quad \forall \mathbf{x} \in D_{\mathbf{R}}^{\operatorname{ar}(f)}\right.
$$

Note that this equation is well-defined since the mapping $d_{\mathbf{v}}$ is injective. Furthermore, we have the following properties.

Lemma 35. (a) $f(\mathbf{y})=f^{\mathbf{v}}\left(d_{\mathbf{v}}(\mathbf{y})\right)$ for any $\mathbf{y} \in D^{\operatorname{ar}(f)}$. (b) $f^{\mathbf{v}}(\mathbf{x})=f(d(\mathbf{x}))$ for $\mathbf{x} \in \operatorname{dom} f^{\mathbf{v}}$.

Finally, we construct the sought language $\Gamma_{\mathbf{R}}$ on domain $D_{\mathbf{R}}$ as follows:

$$
\Gamma_{\mathbf{R}}=\left\{f_{i}^{\mathbf{v}}: i \in[k], \mathbf{v} \in r_{i}\right\} \cup\left\{D_{v}: v \in V\right\}
$$

where relation $D_{v} \subseteq D_{\mathbf{R}}$ is treated as a unary function $D_{v}: D_{\mathbf{R}} \rightarrow\{0, \infty\}$.

Remark 4. We note that there are some parallels between the construction above and the notion of multi-sorted relations [6]. Our approach, however, is different from that in [6]: the language $\Gamma_{\mathbf{R}}$ that we have constructed is a standard (non-multi-sorted) language, which allows us to apply many results known for (V)CSPs.

Remark 5. Lifted language $\Gamma_{\mathbf{R}}$ should not be confused with "G-lifted languages" used in [17]; despite similar names, the constructions are not related.

\subsection{Proof of Proposition 34}

Consider a $\Gamma_{\mathbf{R}}$-instance $\mathcal{I}$ with the set of variables $U$ and the objective function

$$
f_{\mathcal{I}}(h)=\sum_{\left(f_{i}^{\mathbf{v}}, \mathbf{u}\right) \in T} w\left(f_{i}^{\mathbf{v}}, \mathbf{u}\right) f_{i}^{\mathbf{v}}(h(\mathbf{u}))+\sum_{\left(D_{v}, u\right) \in T^{\prime}} w\left(D_{v}, u\right) D_{v}(h(u)) \quad \forall h: U \rightarrow D_{\mathbf{R}}
$$


We can assume w.l.o.g. that each variable $u \in U$ is involved in at least one constraint of arity 2 or higher. (If $u$ is involved in only unary constraints, we can find an optimal solution $h(u)$ independently of other variables, and then remove $u$.) By construction, each constraint induced by a cost function in $\Gamma_{\mathbf{R}}$ restricts each of its variables to a particular copy of $D$ in $D_{\mathbf{R}}$. If different constraints restrict the same variable $u \in U$ to different copies of $D$, then clearly $\mathcal{I}$ has no feasible solutions; we then say that $\mathcal{I}$ is trivially infeasible. Note that we can test this in polynomial time.

Now suppose that $\mathcal{I}$ is not trivially infeasible. Then for each $u \in U$ we can determine in polynomial time node $v \in V$ such that all constraints in $\mathcal{I}$ that involve $u$ restrict solution $h_{u}$ to $D_{v}$. Let $\varphi: U \rightarrow V$ be the corresponding mapping that gives $v=\varphi(u)$. We then have the following property:

Proposition 36. If $\left(f_{i}^{\mathbf{v}}, \mathbf{u}\right) \in T$, then $\mathbf{v}=\varphi(\mathbf{u})$, where $\varphi$ is applied component-wise.

Proof. Let $\mathbf{u}=\left(u_{1}, \ldots, u_{p}\right)$ and $\mathbf{v}=\left(v_{1}, \ldots, v_{p}\right)$. We assumed that the constraint $\left(f_{i}^{\mathbf{v}}, \mathbf{u}\right)$ restricts variable $h\left(u_{j}\right)$ to the domain $D_{\varphi\left(u_{j}\right)}$. By definition of $f_{i}^{\mathbf{v}}$, this function restricts its $j$-th argument to the domain $D_{v_{j}}$. Thus, $\varphi\left(u_{j}\right)=v_{j}$.

Consider an instance $\tilde{\mathcal{I}}$ with the set of variables $U$, the domain $D$ and the cost function

$$
f_{\tilde{\mathcal{I}}}(\tilde{h})=\sum_{\left(f_{i}^{\mathbf{v}}, \mathbf{u}\right) \in T} w\left(f_{i}^{\mathbf{v}}, \mathbf{u}\right) f_{i}(\tilde{h}(\mathbf{u})) \quad \forall \tilde{h}: U \rightarrow D
$$

We claim that solving the instance $\mathcal{I}$ is equivalent to solving the instance $\tilde{\mathcal{I}}$. Indeed, let $\mathcal{S}$ be the set of assignments $h: U \rightarrow D_{\mathbf{R}}$ that are not "trivially infeasible" for $\mathcal{I}$, i.e. that satisfy $h(u) \in D_{\varphi(u)}$ for all $u \in U$. Let $\tilde{\mathcal{S}}$ be the set of assignments $\tilde{h}: U \rightarrow D$. It can be seen that $f_{\mathcal{I}}(h)=\infty$ if $h \notin \mathcal{S}$, and there is a cost-preserving bijection $\tilde{\mathcal{S}} \rightarrow \mathcal{S}$ that maps assignment $\tilde{h} \in \tilde{S}$ to the assignment defined by $h \in \mathcal{S}$ with $h(u)=d_{\varphi(u)}(\tilde{h}(u))$. This implies the claim.

We will show next that $\tilde{\mathcal{I}} \in(\mathrm{V}) \mathrm{CSP}_{\mathcal{H}}(\boldsymbol{\Gamma})$; this will imply the claim of Proposition 34 .

Define relational structure $\tilde{\mathbf{R}}=\left(U, \tilde{r}_{1}, \ldots, \tilde{r}_{k}\right)$ as follows: $\tilde{r}_{i}=\left\{\mathbf{u} \mid\left(f_{i}^{\mathbf{v}}, \mathbf{u}\right) \in T\right\}$ for $i \in[k]$. It defines the set of constraints $\tilde{T}=\left\{\left(f_{i}, \mathbf{u}\right) \mid i \in[k], \mathbf{u} \in \tilde{r}_{i}\right\}=\left\{\left(f_{i}, \mathbf{u}\right) \mid i \in[k],\left(f_{i}^{\mathbf{v}}, \mathbf{u}\right) \in T\right\}$. Using Proposition [36, it can be checked that there is a natural isomorphism between $T$ and $\tilde{T}$, and $\tilde{T}$ defines the set of constraints for the instance $\tilde{\mathcal{I}}$ as in Definition 18. It thus suffices to prove that $\tilde{\mathbf{R}} \in \mathcal{H}$.

We claim that the mapping $\varphi$ is a homomorphism from $\tilde{\mathbf{R}}$ to $\mathbf{R}$. Indeed, we need to show that if $\mathbf{u} \in \tilde{r}_{i}$, then $\varphi(\mathbf{u}) \in r_{i}$. We have $\left(f_{i}^{\mathbf{v}}, \mathbf{u}\right) \in T$ where $\mathbf{v}=\varphi(\mathbf{u})$ by Proposition [36. The condition $f_{i}^{\mathbf{v}} \in \Gamma_{\mathbf{R}}$ implies that $\mathbf{v} \in r_{i}$, or equivalently $\varphi(\mathbf{u}) \in r_{i}$.

We showed that $\tilde{\mathbf{R}} \stackrel{\varphi}{\rightarrow} \mathbf{R}$. Since $\mathbf{R} \in \mathcal{H}$ and $\mathcal{H}$ is up-closed, we obtain that $\tilde{\mathbf{R}} \in \mathcal{H}$, as desired.

\section{Proof of Theorem 23}

We will show the following result.

Proposition 37. Let $\mathcal{H}$ be a structural restriction with $\chi(\mathcal{H})=\infty$ and $\boldsymbol{\Gamma}$ a constraint language of the same signature as $\mathcal{H}$. If for every $\mathbf{R} \in \mathcal{H}$ language $\Gamma_{\mathbf{R}}$ admits a Siggers pair $\left(g_{\mathbf{R}}, s_{\mathbf{R}}\right)$, then $\Gamma$ also admits Siggers pair.

Before giving a proof, we describe how this proposition implies Theorem 23. First, suppose that $\operatorname{CSP}_{\mathcal{H}}(\boldsymbol{\Gamma})$ is tractable. Proposition 34 (a) gives that for every $\mathbf{R} \in \mathcal{H}$ language $\Gamma_{\mathbf{R}}$ is tractable, and thus admits a Siggers pair by Theorem 14. Proposition 37 then gives that $\Gamma$ also admits a Siggers pair, and thus is tractable since $\Gamma$ is a BJK language. 
Now suppose that $\operatorname{CSP}_{\mathcal{H}}(\boldsymbol{\Gamma})$ is not tractable. Then $\Gamma$ is also not tractable, and thus does not admit a Siggers pair (since $\Gamma$ is a BJK language). By Proposition 37 , there exists $\mathbf{R} \in \mathcal{H}$ such that the language $\Gamma_{\mathbf{R}}$ does not admit any Siggers pair, and thus is NP-hard by Theorem 14. Proposition 34(b) now gives that $\mathrm{CSP}_{\mathcal{H}}(\boldsymbol{\Gamma})$ is NP-hard. This also implies NP-hardness of $\operatorname{CSP}(\Gamma)$.

Proof (of Proposition [37). Let $\mathcal{S}$ be the (finite!) set of Siggers pairs $(g, s)$ on the domain $D$. Choose $\mathbf{R} \in \mathcal{H}$ such that $\chi(\mathbf{R})>|\mathcal{S}|$. Let $(g, s)$ be a Siggers pair admitted by $\Gamma_{\mathbf{R}}$. We will use the notation from Sec. 5.1 for the chosen $\mathbf{R}=\left(V, r_{1}, \ldots, r_{k}\right)$. Note that $g$ is a unary operation on $D_{\mathbf{R}}$ and $s$ is a 6-ary operation on $A$, where we denoted $A=g\left(D_{\mathbf{R}}\right) \subseteq D_{\mathbf{R}}$.

For each $v \in V$, we denote the restriction $g_{\mid D_{v}}$ simply as $g_{v}$. The fact $D_{v} \in \Gamma_{\mathbf{R}}$ gives that $g_{v}\left(D_{v}\right) \subseteq D_{v}$. Since this holds for each $v \in V$ and $D_{\mathbf{R}}$ is a disjoint union of $\left\{D_{v}: v \in V\right\}$, we obtain that $g_{v}\left(D_{v}\right)=D_{v} \cap g\left(D_{\mathbf{R}}\right)$. We denote $A_{v}=g_{v}\left(D_{v}\right)=D_{v} \cap A$.

Similarly, we denote the restriction $s_{\mid A_{v}}$ simply as $s_{v}$. We have $D_{v} \in \Gamma_{\mathbf{R}}$ and so $A_{v}=D_{v} \cap A \in$ $\Gamma_{\mathbf{R}}[A]$. Operation $s$ is a polymorphism of $\Gamma_{\mathbf{R}}[A]$, therefore $s_{v}\left(A_{v}, \ldots, A_{v}\right) \subseteq A_{v}$. This shows that $\left(g_{v}, s_{v}\right)$ is a Siggers pair on $D_{v}$. It can also be identified with a Siggers pair on domain $D$ (via a natural isomorphism $\sim_{v}$ induced by the bijection $\left.d: D_{v} \rightarrow D\right)$, and hence we can write $\left(g_{v}, s_{v}\right) \in \mathcal{S}$.

We use the pairs $\left\{\left(g_{v}, s_{v}\right)\right\}_{v \in V}$ to color the elements of $V$. Since $\chi(\mathbf{R})>|\mathcal{S}|$, this coloring is improper and therefore there is a Siggers pair on $D$ ("color") $(\tilde{g}, \tilde{s}) \in \mathcal{S}$ and tuples $\mathbf{v}^{i}=\left(v_{1}^{i}, \ldots, v_{\operatorname{ar}\left(r_{i}\right)}^{i}\right) \in$ $r_{i}$ for each $i \in[k]$ such that $\left(g_{v}, s_{v}\right) \sim_{v}(\tilde{g}, \tilde{s})$ for all $v \in\left\{v_{j}^{i}: i \in[k], j \in\left[\operatorname{ar}\left(r_{i}\right)\right]\right\}$. We will show next that $\Gamma$ admits $(\tilde{g}, \tilde{s})$. We denote $\tilde{A}=\tilde{g}(D) \subseteq D$, then $\tilde{s}$ is a Siggers operation on $\tilde{A}$.

Consider index $i \in[k]$, and let $p$ be the arity of $f_{i}$. We need to show two facts.

- $\tilde{g}$ preserves $f_{i}$. Consider vector $\mathbf{x}=\left(x_{1}, \ldots, x_{p}\right) \in \operatorname{dom} f_{i}$. First, we realize that

$$
\tilde{g}(\mathbf{x})=d\left(g_{v_{1}^{i}}\left(d_{v_{1}^{i}}\left(x_{1}\right)\right), \ldots, g_{v_{p}^{i}}\left(d_{v_{p}^{i}}\left(x_{p}\right)\right)\right)=d(g(\mathbf{y}))
$$

for some $\mathbf{y} \in\left(D_{\mathbf{R}}\right)^{p}$ (namely, $\mathbf{y}=d_{\mathbf{v}^{i}}(\mathbf{x})$ ). Since $\mathbf{x} \in \operatorname{dom} f_{i}$, we also have that $\mathbf{y} \in \operatorname{dom} f_{i}^{\mathbf{v}^{i}}$. As $g$ is a polymorphism of $\Gamma_{\mathbf{R}}$, we get that $g(\mathbf{y}) \in \operatorname{dom} f_{i}^{\mathbf{v}^{i}}$. But this gives $d(g(\mathbf{y})) \in \operatorname{dom} f_{i}$ and we may conclude the proof.

- $\tilde{s}$ preserves $\left(f_{i}\right)_{\mid \tilde{A}}$ (which is the restriction of $f_{i}$ to $\tilde{A}$ ). Let $\mathbf{x}$ be a matrix with $p$ columns (denoted as $\mathbf{x}_{1}, \ldots, \mathbf{x}_{p}$ ) and 6 rows (denoted as $\mathbf{x}^{1}, \ldots, \mathbf{x}^{6}$ ) such that $\mathbf{x}^{1}, \ldots, \mathbf{x}^{6} \in\left[\operatorname{dom} f_{i}\right] \cap \tilde{A}^{p}$. First, we realize that

$$
\tilde{s}\left(\mathbf{x}^{1}, \ldots, \mathbf{x}^{6}\right)=\left(\tilde{s}\left(\mathbf{x}_{1}\right), \ldots, \tilde{s}\left(\mathbf{x}_{p}\right)\right)=d\left(s_{v_{1}^{i}}\left(d_{v_{1}^{i}}\left(\mathbf{x}_{1}\right)\right), \ldots, s_{v_{p}^{i}}\left(d_{v_{p}^{i}}\left(\mathbf{x}_{p}\right)\right)\right)=d\left(s\left(\mathbf{y}^{1}, \ldots, \mathbf{y}^{6}\right)\right)
$$

for some $\mathbf{y}^{1}, \ldots, \mathbf{y}^{6} \in\left(D_{\mathbf{R}}\right)^{p}$ (namely, $\left.\mathbf{y}^{j}=d_{\mathbf{v}^{i}}\left(\mathbf{x}^{j}\right)\right)$. Since $\mathbf{x}^{1}, \ldots, \mathbf{x}^{6} \in\left[\operatorname{dom} f_{i}\right] \cap \tilde{A}^{p}$, we also have that $\mathbf{y}^{1}, \ldots, \mathbf{y}^{6} \in\left[\operatorname{dom} f_{i}^{\mathbf{v}^{i}}\right] \cap A^{p}$. As $s$ is a polymorphism of $\Gamma_{\mathbf{R}}[A]$, we get that $s\left(\mathbf{y}^{1}, \ldots, \mathbf{y}^{6}\right) \in \operatorname{dom} f_{i}^{\mathbf{v}^{i}}$. But this gives $d\left(s\left(\mathbf{y}^{1}, \ldots, \mathbf{y}^{6}\right)\right) \in \operatorname{dom} f_{i}$ and we may conclude the proof.

\section{Proof of Theorem 26}

For a relational structure $\mathbf{R}=\left(V, r_{1}, \ldots, r_{k}\right)$ we define its unary completion $\mathbf{R}^{\prime}$ as follows: take $\mathbf{R}$ and replace every unary relation $r_{i}$ in $\mathbf{R}$ with the unary relation $r_{i}^{\prime}=V$. Since $\mathcal{H}$ does not restrict unaries, we have $\mathbf{R}^{\prime} \in \mathcal{H}$ for each $\mathbf{R} \in \mathcal{H}$. Let $\mathcal{H}^{\prime} \subseteq \mathcal{H}$ be the set of unary-complete relational structures in $\mathcal{H}$, i.e. those structures $\mathbf{R} \in \mathcal{H}$ that satisfy $\mathbf{R}^{\prime}=\mathbf{R}$. It follows from the definition that if a coloring 
$c: V \rightarrow[m]$ is improper for $\mathbf{R}$, then it is also improper for $\mathbf{R}^{\prime}$. Equivalently, if it is proper for $\mathbf{R}^{\prime}$, then it is proper for $\mathbf{R}$. Therefore, $\chi\left(\mathbf{R}^{\prime}\right) \geq \chi(\mathbf{R})$, and consequently $\chi\left(\mathcal{H}^{\prime}\right)=\infty($ since $\chi(\mathcal{H})=\infty)$.

Proposition 38. Suppose that $\mathbf{R}$ is unary-complete relational structure and $\boldsymbol{\Gamma}$ is a conservative valued language. Then there is a polynomial-time reduction from $\operatorname{VCSP}\left(\Gamma_{\mathbf{R}} \cup \Delta_{\mathbf{R}}\right)$ to $\operatorname{VCSP}\left(\Gamma_{\mathbf{R}}\right)$, where $\Delta_{\mathbf{R}}$ is the set of $\{0,1\}$-valued unary functions on the domain $D_{\mathbf{R}}$. Consequently,

(a) if $\operatorname{VCSP}\left(\Gamma_{\mathbf{R}}\right)$ is tractable, then so is $\operatorname{VCSP}\left(\Gamma_{\mathbf{R}} \cup \Delta_{\mathbf{R}}\right)$;

(b) if $\operatorname{VCSP}\left(\Gamma_{\mathbf{R}} \cup \Delta_{\mathbf{R}}\right)$ is $N P$-hard, then so is $\operatorname{VCSP}\left(\Gamma_{\mathbf{R}}\right)$.

Proposition 39. Let $\mathcal{H}^{\prime}$ be a structural restriction with $\chi\left(\mathcal{H}^{\prime}\right)=\infty$ and $\boldsymbol{\Gamma}$ a conservative valued language. If $\operatorname{VCSP}\left(\Gamma_{\mathbf{R}} \cup \Delta_{\mathbf{R}}\right)$ is tractable for every $\mathbf{R} \in \mathcal{H}^{\prime}$, then $\operatorname{VCSP}(\Gamma)$ is tractable.

Let us describe how these propositions imply Theorem 34. First, suppose that $\operatorname{VCSP}_{\mathcal{H}}(\boldsymbol{\Gamma})$ is tractable. Propositions 34(a) and 38(a) give that for every $\mathbf{R} \in \mathcal{H}^{\prime}$ language $\Gamma_{\mathbf{R}} \cup \Delta_{\mathbf{R}}$ is tractable. Thus, by Proposition 39 the language $\Gamma$ is tractable.

Now suppose that $\operatorname{VCSP}_{\mathcal{H}}(\boldsymbol{\Gamma})$ is not tractable. Then $\Gamma$ is also not tractable. By Proposition 39 there exists $\mathbf{R} \in \mathcal{H}^{\prime}$ such that $\Gamma_{\mathbf{R}} \cup \Delta_{\mathbf{R}}$ is not tractable. Language $\Gamma_{\mathbf{R}} \cup \Delta_{\mathbf{R}}$ must then be NP-hard (since it is conservative, and the dichotomy for conservative valued language has been established in [24, see Theorem 42 below). By Propositions 38(b) and 34(b) we obtain that $\operatorname{VCSP}_{\mathcal{H}}(\boldsymbol{\Gamma})$ is NP-hard. This also implies NP-hardness of $\operatorname{VCSP}(\Gamma)$.

\subsection{Proof of Proposition 38}

Consider an instance $\mathcal{I}$ of $\Gamma_{\mathbf{R}} \cup \Delta_{\mathbf{R}}$ with the set of variables $U$ and the objective function

$$
f_{\mathcal{I}}(h)=\sum_{(f, \mathbf{u}) \in T} w(f, \mathbf{u}) f(h(\mathbf{u}))+\sum_{\left(D_{v}, u\right) \in T^{\prime}} w\left(D_{v}, u\right) D_{v}(h(\mathbf{u})) \quad \forall h: U \rightarrow D_{\mathbf{R}}
$$

We can assume w.l.o.g. that each variable $u \in U$ is involved in at least one constraint of arity 2 or higher (by the same argument as in Sec. 5.2). Consider $u \in U$, then by the assumption there exists $v \in V$ such that $h(u) \in D_{v}$ for any feasible assignment $h$. (We use the notation from Sec. 5.1) Let us modify the instance $\mathcal{I}$ by replacing each constraint of the form $(f, u) \in T, f \in \Delta_{\mathbf{R}}$ with $\left(f^{\prime}, u\right)$, where $f^{\prime}: D_{\mathbf{R}} \rightarrow \overline{\mathbb{Q}}$ is defined via

$$
f^{\prime}(x)=\left\{\begin{array}{ll}
f(x) & \text { if } x \in D_{v} \\
\infty & \text { otherwise }
\end{array} \quad \forall x \in D_{\mathbf{R}}\right.
$$

Clearly, this transformation preserves optimal solutions of $\mathcal{I}$. Using the definition of $\Gamma_{\mathbf{R}}$ and the facts that $\mathbf{R}$ is unary-complete and $\boldsymbol{\Gamma}$ is conservative, we conclude that $f^{\prime} \in \Gamma_{\mathbf{R}}$. After applying this transformation for all $u \in U$ with obtain an equivalent instance $\mathcal{I}^{\prime} \in \operatorname{VCSP}\left(\Gamma_{\mathbf{R}}\right)$. This implies the claim.

\subsection{Proof of Proposition 39}

First, we will recall the result on tractability of conservative VCSP languages from [24].

A subset $M \subseteq P$, where $P=\left\{(a, b) \in D^{2}, a \neq b\right\}$ will be called symmetric if $(a, b) \in M$ if and only if $(b, a) \in M$. Sometimes, we will abuse notation slightly by writing $\{a, b\} \in M$.

Definition 40. A fractional operation $\sigma=\frac{1}{2} \chi_{\sqcap}+\frac{1}{2} \chi_{\sqcup}$, where $\sqcap, \sqcup: D^{2} \rightarrow D$, is called a symmetric tournament pair (STP) on symmetric $M \subseteq P$ if both operations $\sqcap$, $\sqcup$ are commutative on $M$, i.e. $a \sqcap b=b \sqcap a$ and $a \sqcup b=b \sqcup a$ for all $(a, b) \in M$, and $(a \sqcap b, a \sqcup b)$ is a permutation of $(a, b)$ for all $(a, b) \in D^{2}$. 
Definition 41. A fractional operation $\mu=\frac{1}{3} \chi_{F_{1}}+\frac{1}{3} \chi_{F_{2}}+\frac{1}{3} \chi_{F_{3}}$, where $F_{1}, F_{2}, F_{3}: D^{3} \rightarrow D$, is called an MJN on symmetric $M \subseteq P$ if $\left(F_{1}(a, b, c), F_{2}(a, b, c), F_{3}(a, b, c)\right)$ is a permutation of $(a, b, c)$ for $a, b, c \in D$ and if whenever $\{a, b, c\}=\{x, y\}$ for some $\{x, y\} \in M$ then $F_{1}(a, b, c)=F_{2}(a, b, c)$ is the unique majority element among $a, b, c$ (that occurs twice) and $F_{3}(a, b, c)$ is the unique minority element among $a, b, c$ (that occurs once).

Theorem 42 (24]). Let $\Gamma$ be a conservative valued language and $P=\left\{(a, b) \in D^{2}, a \neq b\right\}$. If there is a symmetric set $M^{\prime} \subseteq P$ such that $\Gamma$ admits an $S T P$ on $M^{\prime}$ and an MJN on $P \backslash M^{\prime}$ as fractional polymorphisms, then $\operatorname{VCSP}(\Gamma)$ is tractable. Otherwise, $\operatorname{VCSP}(\Gamma)$ is $N P$-hard.

Now we are ready to prove Proposition 39 and thus conclude the proof of Theorem 26.

Proof. Let $\mathcal{S}$ be the (finite!) set

$$
\{(\sigma, \mu, M): \sigma \text { is } \mathrm{STP} \text { on } M, \mu \text { is MJN on } P \backslash M, M \subseteq P \text { symmetric }\} \text {. }
$$

Choose $\mathbf{R}=\left(V, r_{1}, \ldots, r_{k}\right) \in \mathcal{H}^{\prime}$ such that $\chi(\mathbf{R})>|\mathcal{S}|$. Since $\operatorname{VCSP}\left(\Gamma_{\mathbf{R}} \cup_{\mathbf{R}}\right)$ is tractable and the language is conservative, Theorem 42 gives us a symmetric subset $M_{\mathbf{R}} \subseteq P_{\mathbf{R}}$, where $P_{\mathbf{R}}=\{(a, b) \in$ $\left.D_{\mathbf{R}}^{2}, a \neq b\right\}$, an STP $\sigma=\frac{1}{2} \chi_{\sqcap}+\frac{1}{2} \chi_{\sqcup}$ on $M_{\mathbf{R}}$, and an MJN $\mu=\frac{1}{3} \chi_{F_{1}}+\frac{1}{3} \chi_{F_{2}}+\frac{1}{3} \chi_{F_{3}}$ on $P_{\mathbf{R}} \backslash M_{\mathbf{R}}$, such that both $\sigma$ and $\mu$ are fractional polymorphisms of $\Gamma_{\mathbf{R}} \cup \Delta_{\mathbf{R}}$.

For each $v \in V$, we define a symmetric $M_{v} \subseteq P$ as

$$
M_{v}=\left\{(a, b) \in P:\left(d_{v}(a), d_{v}(b)\right) \in M_{\mathbf{R}}\right\} .
$$

(Again, we use the notation from Sec. 5.1) Further, we set $\sigma_{v}=\frac{1}{2} \chi_{\sqcap_{v}}+\frac{1}{2} \chi_{\sqcup_{v}}$ where $\sqcap_{v}: D^{2} \rightarrow D$ is given by $a \sqcap b=d\left(d_{v}(a) \sqcap d_{v}(b)\right)$ and $\sqcup_{v}: D^{2} \rightarrow D$ is defined analogously. And finally $\mu_{v}=$ $\frac{1}{3} \chi_{F_{1 v}}+\frac{1}{3} \chi_{F_{2 v}}+\frac{1}{3} \chi_{F_{3 v}}$, where for $i=1,2,3$ we define $F_{i v}: D^{3} \rightarrow D$ as

$$
F_{i v}(a, b, c)=d\left(F_{i}\left(d_{v}(a), d_{v}(b), d_{v}(c)\right)\right) .
$$

It is easily seen that $\sigma_{v}$ is an STP on $M_{v}$ and $\mu_{v}$ is an MJN on $P \backslash M_{v}$ and thus $\left(\sigma_{v}, \mu_{v}, M_{v}\right) \in \mathcal{S}$.

We use the triples $\left(\sigma_{v}, \mu_{v}, M_{v}\right)$ as colors for elements of $V$. Since $\chi(\mathbf{R})>|\mathcal{S}|$, this coloring is improper and therefore there exists a triple $(\tilde{\sigma}, \tilde{\mu}, \tilde{M}) \in \mathcal{S}$ and tuples $\mathbf{v}^{i}=\left(v_{1}^{i}, \ldots, v_{\operatorname{ar}\left(r_{i}\right)}^{i}\right) \in r_{i}$ for each $i \in[k]$ such that $\left(\sigma_{v}, \mu_{v}, M_{v}\right)=(\tilde{\sigma}, \tilde{\mu}, \tilde{M})$ for all $v \in\left\{v_{j}^{i}: i \in[k], j \in\left[\operatorname{ar}\left(r_{i}\right)\right]\right\}$.

Next, we show that $\tilde{\sigma}$ and $\tilde{\mu}$ are fractional polymorphisms of $\Gamma$. This finishes the proof, since then $\operatorname{VCSP}(\Gamma)$ is tractable by Theorem 42 .

Let us show that $\tilde{\sigma}=\frac{1}{2} \chi_{\tilde{\Gamma}}+\frac{1}{2} \chi_{\tilde{\cup}}$ is admitted by a cost function $f_{i} \in \Gamma$ for $i \in[k]$. Take $\mathbf{a}=\left(a_{1}, \ldots, a_{p}\right), \mathbf{b}=\left(b_{1}, \ldots, b_{p}\right) \in \operatorname{dom} f_{i}$ where $p$ is the arity of $f_{i}$. First note that

$$
\mathbf{a} \tilde{\sqcap} \mathbf{b}=\left(a_{1} \sqcap_{v_{1}^{i}} b_{1}, \ldots, a_{p} \sqcap_{v_{p}^{i}} b_{p}\right)=\left(d\left(d_{v_{1}^{i}}\left(a_{1}\right) \sqcap d_{v_{1}^{i}}\left(b_{1}\right)\right), \ldots, d\left(d_{v_{p}^{i}}\left(a_{p}\right) \sqcap d_{v_{p}^{i}}\left(b_{p}\right)\right)\right) .
$$

Therefore we get

$$
f_{i}(\mathbf{a} \tilde{\sqcap} \mathbf{b})=f_{i}\left(d\left(d_{v_{1}^{i}}\left(a_{1}\right) \sqcap d_{v_{1}^{i}}\left(b_{1}\right)\right), \ldots, d\left(d_{v_{p}^{i}}\left(a_{p}\right) \sqcap d_{v_{p}^{i}}\left(b_{p}\right)\right)\right)=f_{i}^{\mathbf{v}^{i}}\left(d_{\mathbf{v}^{i}}(\mathbf{a}) \sqcap d_{\mathbf{v}^{i}}(\mathbf{b})\right)
$$

where in the second equality we used Lemma 35. Since we have similar equalities for $\tilde{\sqcup}$ and since $f_{i}^{\mathbf{v}^{i}} \in \Gamma_{\mathbf{R}}$ admits $\sigma$, we get the sought

$$
\begin{aligned}
f_{i}(\mathbf{a} \tilde{\sqcap} \mathbf{b})+f_{i}(\mathbf{a} \tilde{\sqcup} \mathbf{b}) & =f_{i}^{\mathbf{v}^{i}}\left(d_{\mathbf{v}^{i}}(\mathbf{a}) \sqcap d_{\mathbf{v}^{i}}(\mathbf{b})\right)+f_{i}^{\mathbf{v}^{i}}\left(d_{\mathbf{v}^{i}}(\mathbf{a}) \sqcup d_{\mathbf{v}^{i}}(\mathbf{b})\right) \\
& \leq f_{i}^{\mathbf{v}^{i}}\left(d_{\mathbf{v}^{i}}(\mathbf{a})\right)+f_{i}^{\mathbf{v}^{i}}\left(d_{\mathbf{v}^{i}}(\mathbf{b})\right)=f_{i}(\mathbf{a})+f_{i}(\mathbf{b})
\end{aligned}
$$

where in the last equality we used Lemma 35. Hence $\tilde{\sigma}$ is admitted by $\Gamma$ and for analogous reasons also $\tilde{\mu}$ is admitted by $\Gamma$. 


\section{Acknowledgements}

We thank Andrei Krokhin for helpful comments on the manuscript. This work was supported by the European Research Council under the European Unions Seventh Framework Programme (FP7/20072013)/ERC grant agreement no 616160.

\section{References}

[1] K. Appel and W. Haken. Every planar map is four colorable. Part i: Discharging. Illinois J. Math., 21(3):429-490, 091977.

[2] L. Barto and M. Kozik. New conditions for Taylor varieties and CSP. In Proceedings of the 25th Annual IEEE Symposium on Logic in Computer Science, LICS 2010, 11-14 July 2010, Edinburgh, United Kingdom, pages 100-109, 2010.

[3] L. Barto, M. Kozik, and T. Niven. The CSP dichotomy holds for digraphs with no sources and no sinks (a positive answer to a conjecture of Bang-Jensen and Hell). SIAM Journal on Computing, 38(5):1782-1802, 2009.

[4] A. Bulatov. A dichotomy theorem for constraint satisfaction problems on a 3-element set. Journal of the ACM, 53(1):66-120, 2006.

[5] A. Bulatov. Complexity of conservative constraint satisfaction problems. ACM Transactions on Computational Logic, 12(4), 2011. Article 24.

[6] A. Bulatov and P. Jeavons. An algebraic approach to multi-sorted constraints. In $C P^{\prime} 03$, volume 2833 of $L N C S$, pages 183-198, 2003.

[7] A. Bulatov, A. Krokhin, and A. Jeavons. Classifying the Complexity of Constraints using Finite Algebras. SIAM Journal on Computing, 34(3):720-742, 2005.

[8] J. Bulín, D. Delic, M. Jackson, and T. Niven. On the reduction of the CSP dichotomy conjecture to digraphs. In Christian Schulte, editor, CP, volume 8124 of Lecture Notes in Computer Science, pages 184-199. Springer, 2013.

[9] D. Cohen, M. Cooper, and P. Jeavons. An algebraic characterisation of complexity for valued constraints. In CP'06, volume 4204 of $L N C S$, pages 107-121, 2006.

[10] D. Cohen, M. C. Cooper, P. Jeavons, and A Krokhin. The Complexity of Soft Constraint Satisfaction. Artificial Intelligence, 170(11):983-1016, 2006.

[11] David A. Cohen, Martin C. Cooper, Páidí Creed, Peter Jeavons, and Stanislav Živný. An algebraic theory of complexity for discrete optimisation. SIAM Journal on Computing, 2013.

[12] Stephen A. Cook. The complexity of theorem-proving procedures. In Proceedings of the Third Annual ACM Symposium on Theory of Computing, STOC '71, pages 151-158, New York, NY, USA, 1971. ACM.

[13] Martin C. Cooper and Stanislav Živný. Hybrid tractability of valued constraint problems. Artificial Intelligence, 175(9-10):1555-1569, 2011.

[14] P. Erdős. On the construction of certain graphs. Journal of Combinatorial Theory, 1(1):149 $153,1966$. 
[15] Tomás Feder and Moshe Y. Vardi. The Computational Structure of Monotone Monadic SNP and Constraint Satisfaction: A Study through Datalog and Group Theory. SIAM Journal on Computing, 28(1):57-104, 1998.

[16] David Geiger. Closed systems of functions and predicates. Pacific J. Math., 27(1):95-100, 1968.

[17] Martin J. Green and David A. Cohen. Domain permutation reduction for constraint satisfaction problems. Artificial Intelligence, 172(8-9):1094-1118, 2008.

[18] Martin Grohe. The complexity of homomorphism and constraint satisfaction problems seen from the other side. J. ACM, 54(1):1:1-1:24, March 2007.

[19] M. Grötschel, L. Lovász, and A. Schrijver. Geometric Algorithms and Combinatorial Optimization. Springer-Verlag, New York, 1988.

[20] Pavol Hell and Jaroslav Nešetřil. On the complexity of h-coloring. Journal of Combinatorial Theory, Series B, 48(1):92 - 110, 1990.

[21] P. Jeavons, A. Krokhin, and S. Živný. The complexity of valued constraint satisfaction. Bulletin of the EATCS, 113:21-55, 2014.

[22] Peter Jeavons. On the algebraic structure of combinatorial problems. Theor. Comput. Sci., 200(1-2):185-204, June 1998.

[23] Philippe Jégou. Decomposition of domains based on the micro-structure of finite constraintsatisfaction problems. In Richard Fikes and Wendy G. Lehnert, editors, AAAI, pages 731-736. AAAI Press / The MIT Press, 1993.

[24] V. Kolmogorov and S. Živný. The complexity of conservative valued CSPs. Journal of the ACM, 60(2), 2013. Article 10.

[25] A. V. Kuznetsov. Algebra of logic and their generalizations. In S. Janovskaya, editor, Mathematics in USSR for 40 years, volume 1, pages 105-115. Fizmatgiz Moscow, 1959.

[26] W. Mader. Homomorphieeigenschaften und mittlere kantendichte von graphen. Mathematische Annalen, 174(4):265-268, 1967.

[27] M. Maróti and R. McKenzie. Existence theorems for weakly symmetric operations. Algebra universalis, 59(3-4):463-489, October 2008.

[28] J. Nešetřil and P. Ossona de Mendez. Colorings and homomorphisms of minor closed classes. In Boris Aronov, Saugata Basu, János Pach, and Micha Sharir, editors, Discrete and Computational Geometry, volume 25 of Algorithms and Combinatorics, pages 651-664. Springer Berlin Heidelberg, 2003.

[29] Emil L. Post. On The Two-Valued Iterative Systems of Mathematical Logic. Princeton University Press, 1941.

[30] T. J. Schaefer. The Complexity of Satisfiability Problems. In Proceedings of the 10th Annual ACM Symposium on Theory of Computing (STOC'78), pages 216-226. ACM, 1978.

[31] M. H. Siggers. A strong Mal'cev condition for locally finite varieties omitting the unary type. Algebra universalis, 64(1-2):15-20, October 2010. 
[32] Jacobus Stephanus Swarts. The complexity of digraph homomorphisms: Local tournaments, injective homomorphisms and polymorphisms. PhD thesis, University of Victoria, Canada, 2008.

[33] Rustem S. Takhanov. A dichotomy theorem for the general minimum cost homomorphism problem. In In Proceedings of the 27th International Symposium on Theoretical Aspects of Computer Science (STACS), pages 657-668, 2010.

[34] J. Thapper and S. Živný. Sherali-Adams relaxations for valued CSPs. Technical report, arXiv:1502.05301, 2015.

[35] Johan Thapper and Stanislav Živný. The complexity of finite-valued CSPs. In Proceedings of the 45th ACM Symposium on the Theory of Computing (STOC'13), pages 695-704. ACM, 2013. 